\title{
A Graphene THz Detector based on Plasmon Resonances and Interband Transitions
}

\author{
A. Maffucci*(1), S.A. Maksimenko ${ }^{(2)}$, M.E. Portnoi ${ }^{(3)}$, V.A. Saroka ${ }^{(2),(4)}$, and G.Y. Slepyan ${ }^{(5)}$
}

(1) Dep. DIEI, University of Cassino and Southern Lazio, Cassino, 03043 Italy (maffucci@unicas.it)

(2) INP, Belarusian State University, Bobruiskaya 11, 220030, Minsk, Belarus

(3) School of Physics, University of Exeter, Stocker Road, Exeter EX4 4QL, UK

(4) Center for Quantum Spintronics, Dep. of Physics, Norwegian Univ. of Science and Techn., NO-7491 Trondheim, Norway

(5) School of Electrical Engineering, Tel Aviv University, Tel Aviv, 6997801, Israel

\begin{abstract}
A novel terahertz detector is here proposed, based on the use of planar arrays of graphene nanoribbons of different widths and lengths. Two distinct mechanisms are exploited to detect an external incident field: the excitation of interband transitions and of the surface plasmon polaritons resonances. The tuning of such mechanisms allows strengthening the signal, so providing an alternative solution to the problem of the low efficiency of the resonant graphene antennas. This paper proposes a possible design solution and analyzes and the feasibility of tuning the two mechanisms in the $\mathrm{THz}$ range.
\end{abstract}

\section{Introduction}

The Terahertz (THz) range technology is expected to bring unprecedented enhancements in imaging, spectroscopy and communication applications in a wide range of fields, from medicine to security, from astrophysics to consumer electronics [1]. In such a range, conventional solutions are not efficient, being such frequencies too high for the electronics/microwave technology and too low for the photonics/infrared (IR) one. Anyway, conventional detectors have been realized based on heterojunction backward diodes [2] and Schottky diodes [3]. The recent advances in nanotechnology opened an alternative route to the $\mathrm{THz}$ technology, exploiting the novel electromagnetic behavior of nanomaterials, such as the graphene-related materials, e.g., carbon nanotubes (CNTs) or graphene nanoribbons (GNRs). Their high carrier mobility, frequency-independent absorption and gapless spectrum, can lead to $\mathrm{THz}$ devices more compact and efficient than those based on conventional materials [4].

In this paper, we focus on $\mathrm{THz}$ detectors based on the use of graphene nanoribbons as antenna elements. Many mechanisms can be envisaged to use such elements for detecting a $\mathrm{THz}$ wave impinging on them: in [5] the proposed detector is based on the excitation of interband transitions between multiple graphene layers. In [6] a detector is proposed by using antenna coupling with a graphene FET, exploiting the non-linear response. The use of tunneling resonances is another way to follow [7].
Anyway, as far as now, the most promising mechanism seems to be the excitation on graphene layers of the socalled Surface Plasmon Polaritons (SPP), i.e. highly confined modes in the IR and THz range [8]. An advantage in using graphene-related materials is the possibility of provide a tuning via electrical and magnetic doping. On the other side, a major drawback of SPP detectors is the low efficiency of the coupling between the incident $\mathrm{THz}$ radiation and the detector itself. Solutions so far proposed to enhance the performance (for instance, in terms of the strength of the signal level) are based on the use of multilayered structures [9]-[10]. However, from the technological point of view, the realization of nanoscale stacks of graphene/insulator or graphene/metal layers is a major challenge, given the present status of the manufacturing technology of graphene devices.

In this paper, we propose a solution that could enhance the performance of a planar graphene detector without moving to a 3D stacked architecture. The detector module is a planar array of graphene ribbons of variable widths and lengths, as depicted in Fig.1. In the next Sections, it is demonstrated that an accurate design of the lengths and widths allows tuning the resonances coming from two different mechanisms: excitation of SPPs and of interband transitions. The proposed planar solution is not only simpler to be realized compared to the stacked one, but it also makes easier the handling of electric contacts and the back-gate control of the Fermi level position, to be placed within the bandgap.

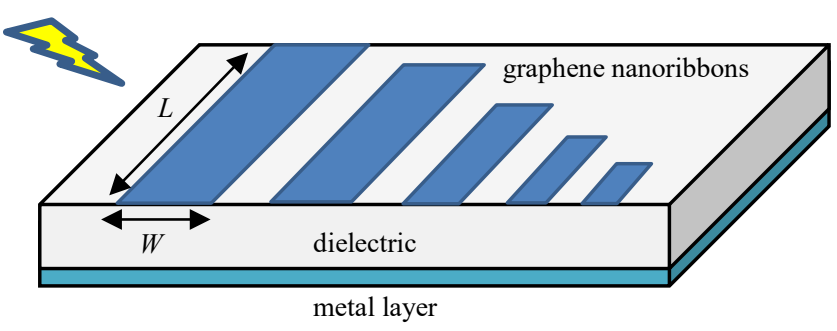

Figure 1. The element of the proposed broadband $\mathrm{THz}$ detector: an array of graphene ribbons of diffent lengths and widths lying on a dielectric layer. 


\section{SPP resonances and interband transitions}

The existence of the Surface Plasmon Polaritons (SPPs) is related to the quantum nature of the electrodynamics of carbon nanostructures and can be theoretically demonstrated by evaluating their electrical conductivity taking into account both intraband and interband transitions, as done, for instance, in [11] for CNTs. Let us consider a graphene ribbon of length $L$ and width $W$ as in Fig.1, denoting with $\sigma$ is its conductivity. The ribbon is placed on a dielectric substrate of relative permittivity $\varepsilon_{r}$, with air above, of permittivity $\varepsilon_{0}$. This structure can support SPP waves, whose dispersion relation may be derived by using a Fabry-Perot model as follows:

$$
\frac{1}{\sqrt{\left(\frac{\beta}{k_{0}}\right)^{2}-1}}+\frac{\varepsilon_{r}}{\sqrt{\left(\frac{\beta}{k_{0}}\right)^{2}-\varepsilon_{r}}}=-i \frac{\sigma(\omega)}{\omega \varepsilon_{0}} k_{0},
$$

where $k_{0}$ the free-space wavenumber, $\beta$ and $\omega$ are the wave-vector and frequency of the SPP wave. Imposing in (1) the classical antenna resonance condition

$$
L=n \frac{\lambda}{2}=n \frac{\pi}{\beta}, \quad n=1,2.3 \ldots
$$

would provide a series of complex frequencies $\omega_{n}$, associated to the existing SPP resonant modes. From (1) and (2) it results that the position of the resonances in the frequency spectrum can be tuned by the length of the ribbon, as in the classical antennas. Note that the conductivity $\sigma$ depends on $L$ and on $W$, hence the SPP resonance frequency will also depend on $W$.

In graphene related materials, the phase velocity $v_{p h}=\beta c$ is much smaller than the speed of light in vacuum (about two orders of magnitude) [8]: this strong slowing-down of the waves is responsible for the possibility of having antenna resonances in the $\mathrm{THz}$ or IR ranges associated to the effect of finite length, for lengths values in the submicron range.

Interband transitions may be induced in GNRs as well as in CNTs which have narrow energy gaps in the $\mathrm{THz}$ range. The probability of the interband dipole transition is proportional to the squared matrix element of the velocity operator calculated between the corresponding states [12].This sharp peak in the $\mathrm{THz}$ transition matrix element in conjunction with the van Hove singularity in the quasione-dimensional GNR spectrum leads to high sensitivity of the photocurrent to the excitation frequency, which is at the basis of the proposed detector. An additional control of the GNR band gap can be achieved by applying an in-plane electric field

\section{Results and discussion}

Let us start investigating the interband transitions related to the nanoribbons in Fig.1, for varying values of the width $W$. In the following, we will assume armchair GNRs (aGNRs), whose width is given by $W=N b$, being $b=$
$0.142 \mathrm{~nm}$ and $N=3 \mathrm{p}+2$, where $N$ is the number of dimers in W ( $p$ is an integer). By using Eq. (10) from Ref. [13] with third order hopping integral $t_{3}=-0.3 \mathrm{eV}$ and edge correction $\mathrm{dt}=-0.2 \mathrm{eV}$ we can estimate the frequency of the lowest energy interband transitions occurring in such a family of GNRs. The results are presented in Fig. 2 and refer to an infinitely-long GNRs. The matrix element of these transitions has a universal value and equal to the Fermi velocity in graphene [12],[14]. In order to decouple the interband transition phenomenon from the plasmon resonance one, it is useful to stress that the length $L$ of the GNRs does not have much effect on the absorption spectrum for high aspect ratio values, such as $L / W>30$ [15].

Let us now investigate the SPP resonance values that we can obtain from (1) and (2). An approximated expression for the conductivity of a-GNRs is [16]:

$$
\sigma(\omega)=\frac{2 v_{F} M(W, T)}{\pi v R_{0} W} \frac{1}{1+i \omega / v}
$$

where $M$ is the equivalent number of conducting channels (which depends on the ribbon chirality, width and temperature $T$ ), $\quad R_{0}=12.9 \mathrm{k} \Omega$ is the quantum resistance, $v$ is the collision frequency, $v_{F}$ is the Fermi velocity. Assuming $T=300 \mathrm{~K}$ and evaluating numerically $M$ as indicated in [16], the conductivity (3) can be used in (1) along with condition (2), to obtain the SPP resonances. The first plasmon resonance is plotted in Fig.3, as a function of $L$, assuming a silicon substrate with $\varepsilon_{r}=12$, and assuming for the GNR the following typical values: $v_{F}=8.7 \cdot 10^{5} \mathrm{~m} / \mathrm{s}$, and $v=1 \cdot 10^{12} \mathrm{THz}$. Note that for these values, the first SPP resonance does not significantly depend on $W$, since the variation of the conductivity (3) on $W$ has a slight effect on the dispersion relation (1). Therefore, for these values, the study of the SPP resonance results to be decoupled from that of the interband transitions. By comparing the results in Fig.2 and Fig.3, it is clear that there are ranges of values where it is possible to excite both the mechanisms. Table I reports the dimensions requested to a GNR to have at the same time SPP resonance and interband transition, at the given frequencies. Note that the aspect ratio is always $>60$.

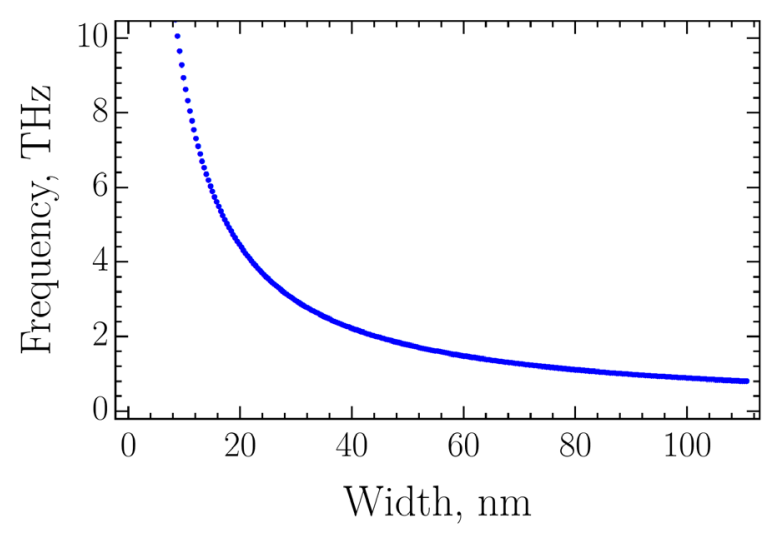

Figure 2. Frequency of the interband optical transition across the narrow band gap of an-infinitely long armchair GNRs, versus the width $W$. 


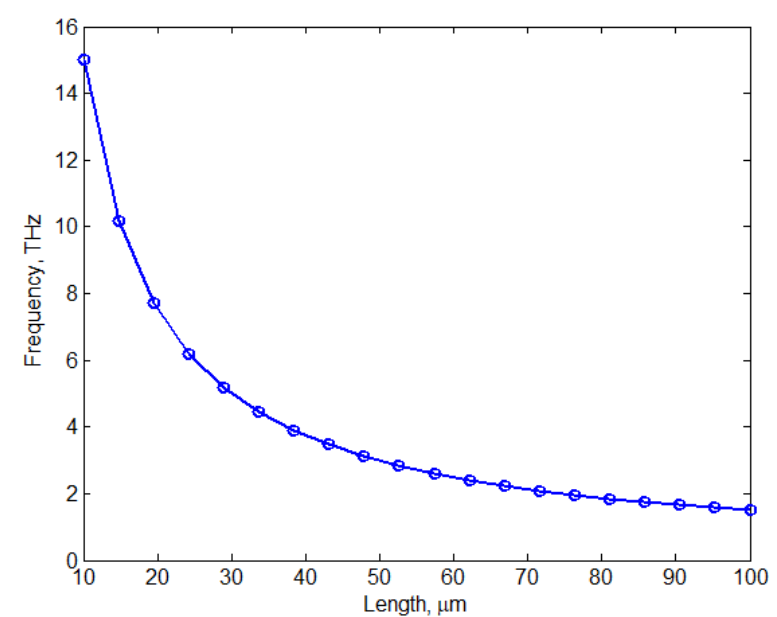

Figure 3. First plasmon resonance associated to an element of the detector in Fig. 1 for varying values of $L$.

Further work will be devoted to the calculation of the antenna efficiency in the $\mathrm{THz}$ range, as a consequence of the effects of the two mechanisms. Knowing the efficiency, one may use the present result in two ways: (i) the design in Fig. 1 can be made with GNRs with equal $W$ and $L$, to realize a narrow-band detector but with increased signal strength; (ii) the design can be made by GNRs with different $W$ and $L$, to realize a broadband detector.

TABLE I

WIDTHS AND LENGTHS FOR THE GNRS IN FIG.1 AND CORRESPONDING RESONANCE FREQUENCIES

\begin{tabular}{ccc}
\hline $\begin{array}{c}\text { FREQUENCY } \\
{[\mathrm{THz}]}\end{array}$ & $\begin{array}{c}\text { WIDTH } \\
{[\mathrm{nm}]}\end{array}$ & $\begin{array}{c}\text { LENGTH } \\
{[\mu \mathrm{m}]}\end{array}$ \\
\hline 2.00 & 44 & 76.3 \\
\hline 4.00 & 24 & 37.2 \\
\hline 6.00 & 12 & 24.6 \\
\hline 8.00 & 15 & 19.1 \\
\hline 10.00 & 8 & 14.3 \\
\hline \hline
\end{tabular}

\section{Acknowledgements}

This work was supported by the Project EU-H2020MSCA-RISE “TERASSE”, Grant n. 823878 (2019-2023).

\section{References}

1. T. Nagatsuma, "Terahertz technologies: present and future," IEICE Electronics Express, 8, 14, 2011, pp. 11271142, doi: 10.1587/elex.8.1127.

2. Z. Zhang, R. Rajavel, P. Deelman, P. Fay, "Sub-micron area heterojunction Backward Diode Millimeter-Wave Detectors With $0.18 \mathrm{pW} / \mathrm{Hz}^{1} / 2$ Noise Equivalent Power," IEEE Microw. and Wireless Comp. Letters, 21, 5, 2011, pp. 267-269, doi: 10.1109/LMWC.2011.2123878.

3. E. Schlecht, J. V. Siles, C. , R. Lin, B. Thomas, "Schottky Diode Based 1.2 THz Receivers Operating at
Room-Temperature and Below for Planetary Atmospheric Sounding," IEEE Trans. on Terahertz Science and Techn., 4, 6, 2014, pp.661-669, doi:10.1109/TTHZ.2014.2361621.

4. R.R. Hartmann, J. Kono, M.E. Portnoi, "Terahertz science and technology of carbon nanomaterials," Nanotechnology, 25, 2014, p.322001, doi: 10.1088/0957$4484 / 25 / 32 / 322001$.

5. V. Ryzhii, M. Ryzhii, N. Ryabova, V. Mitin, T. Otsuji, "Terahertz and infrared detectors based on graphene structures," Infrared Physics \& Technology, 54, 2011, pp. 302-305, doi: 10.1016/j.infrared.2010.12.034.

6. L. Vicarelli, et al., "Graphene field-effect transistors as room temperature terahertz detectors," Nature materials, 11, 2012, pp.865-871.

7. A. Maffucci, "A New Mechanism for $\mathrm{THz}$ Detection Based on the Tunneling Effect in Bi-Layer Graphene Nanoribbons," Applied Sciences, 5, 2015, 1102-116, doi: 10.3390/app5041102

8. A. Maffucci, S. Maksimenko, "Carbon-based terahertz resonant antennas," in Fundamental nanoelectromagnetics II, Springer, 2019, ISBN: 9789402416862

9. D.A. Bandurin et al. "Resonant terahertz detection using graphene plasmons," Nature Communications 9, 2018, p. 5392, doi: 10.1038/nmat3417.

10. Y. Huang, S. Zhong, H. Yao, and D. Cui, "Tunable Terahertz Plasmonic Sensor Based on Graphene/Insulator Stacks," IEEE Photonics Journal, 9, 1, 2017, p.5900210, doi: 10.1109/JPHOT.2017.2656242.

11. G.Y. Slepyan, S.A. Maksimenko, et al., "Electrodynamics of carbon nanotubes: Dynamics conductivity, impedance boundary conditions, and surface wave propagation," Phys Rev B, 60, 1999, p.17136, doi: 10.1103/PhysRevB.60.17136.

12. R. R. Hartmann, V. A. Saroka, and M. E. Portnoi, "Interband transitions in narrow-gap carbon nanotubes and graphene nanoribbon," Journ. Applied Physics, 125, 2019, p.121607, doi: 10.1063/1.5080009.

13. D. Gunlycke, C.T. White, "Tight-binding energy dispersions of armchair-edge graphene nanostrips," Physical Review B, 77, 2008, p.115116 doi: 10.1103/PhysRevB.77.115116.

14. M. E. Portnoi, V. A. Saroka, R. R. Hartmann, and O. V. Kibis, "Carbon Nanotubes and Graphene Nanoribbons," in Proc. of 2015 IEEE Comput. Soc. Annu. Symp. VLSI, Montpellier (FR) 2015, pp. 456-459.

15. V.A. Saroka, A.L. Pushkarchuk, S.A. Kuten, and M. E. Portnoi, "Hidden correlation between absorption peaks in achiral carbon nanotubes and nanoribbons," J. Saudi, Chem. Soc., 22, 2018, 985, doi:10.1016/j.jscs.2018.03.001

16. A. Maffucci, G. Miano, "Number of Conducting Channels for Armchair and Zig-Zag Graphene Nanoribbon Interconnects," IEEE Trans. on Nanotechnology, 12, 2013, pp. 817-823, doi: 10.1109/TNANO.2013.2274901. 